K. ATSUYAMA

KODAI MATH. J.

6 (1983), 122-133

\title{
ANOTHER CONSTRUCTION OF REAL SIMPLE LIE ALGEBRAS
}

\author{
By Kenji Atsuyama
}

\section{Introduction.}

In 1966, J. Tits [6] and E. B. Vinberg [7] made explicit models of exceptional simple Lie algebras independently. It is known that Tits' models contain all the real forms of these Lie algebras (cf. N. Jacobson [4]). In this paper we shall give first another construction of compact (real) simple Lie algebras which are isomorphic to Tits' and Vinberg's models in the case of exceptional Lie algebras. Furthermore we shall make all involutive automorphisms in each Lie algebra and also give all the real forms explicitly, corresponding to the involutive automorphisms, which are simpler than Tits' Lie algebras.

\section{Preliminaries.}

Let $\mathfrak{A}$ be a composition algebra over the field $\boldsymbol{R}$ of real numbers. Let $a, b, c$ be elements in $\mathfrak{A}$. If a conjugation $-: a \rightarrow \vec{a}$ is usually defined in $\mathfrak{A}$, we have a symmetric inner product $(a, b)=1 / 2(a b+\overline{a b})$. If the commutator and the associator are written as $[a, b]=a b-b a$ and $(a, b, c)=(a b) c-a(b c)$ respectively, any inner derivation of $\mathfrak{A}$ can be generated by $D_{a, b}$ where $D_{a, b}(c)=[[a, b], c]$ $-3(a, b, c)$.

In the composition algebra $\mathfrak{A}$, it is well known that the following identities hold (cf. R. D. Schafer [5]).

LEMMA 1.1. For $a, b, c \in \mathfrak{A}$, we have that

(1) $(a b, c)=(b c, a)$,

(2) $(a, b, c)=(b, c, a)=-(b, a, c)$,

(3) $D_{a, b}=-D_{b, a}, \overline{D_{a, b}(c)}=D_{\bar{a}, \bar{b}}(\bar{c})$,

(4) $(D a, b)+(a, D b)=0$,

(5) $\left[D, D_{a, b}\right]=D_{D a, b}+D_{a, D b}$,

(6) $D_{a b, c}+D_{b c, a}+D_{c a, b}=0$,

where $D$ is any inner derivation of $\mathfrak{A}$.

Let $M(n, \boldsymbol{R})$ denote an $n \times n$ matrix algebra over $\boldsymbol{R}$ with coefficients in $\boldsymbol{R}$. Let $X, Y$ be elements in $M(n, \boldsymbol{R})$. We usually define a transposed operator

Received June 2, 1982 
$T: X \rightarrow X^{T}$ in $M(n, \boldsymbol{R})$ and define a symmetric inner product by $(X, Y)=\operatorname{tr}(X Y)$ where $\operatorname{tr}(X)=1 / n\left(x_{11}+\cdots+x_{n n}\right)$ for $X=\left(x_{\imath \jmath}\right) \in M(n, \boldsymbol{R})$. Then we have $(X Y, Z)$ $=(Y Z, X)$.

Let $X, Y$ and $Z$ be in $M(3, \boldsymbol{R})$. If $f(X, Y, Z)$ is a polynomial of $X, Y$ and $Z$, then $\Sigma f(X, Y, Z)$ means the sum over all permutations of $(X, Y, Z)$ relative to $f(X, Y, Z)$ and $\subseteq f(X, Y, Z)$ means the sum over cyclic permutations of $(X, Y, Z)$.

In $M(3, \boldsymbol{R})$ we can prove the following proposition by direct calculations.

Proposition 1.2. For $X, Y, Z \in M(3, \boldsymbol{R})$, we have the following identities where $I$ is the $3 \times 3$ unit matrix.

(1) If $X, Y$ and $Z$ are symmetric matrices with the trace being 0 ,

$$
\Sigma X Y Z-3 \Subset(X, Y) Z-6 \operatorname{tr}(X Y Z) I=0 \text { 。 }
$$

(2) If $X$ and $Y$ are skew-symmetruc matrices,

$$
2 X Y X=3(X, Y) X \text {. }
$$

(3) If $Y$ and $Z$ are skew-symmetruc matrices and $X$ is a symmetruc matrix with the trace being 0 ,

$$
2(X Y Z+Y Z X+Y X Z)-3(Y, Z) X-6 \operatorname{tr}(X Y Z) I=0 .
$$

(4) If $X$ is a symmetric matrix with the trace being 0 and $Y$ is a skewsymmetric matrix,

$$
2\left(X^{2} Y+X Y X+Y X^{2}\right)=3(X, X) Y
$$

Let $\mathfrak{A}^{(1)} \otimes M(n, \boldsymbol{R}) \otimes \mathfrak{U}^{(2)}$ be a tensor product over $\boldsymbol{R}$ of one matrix algebra and two composition algebras. We write $a X u$ instead of $a \otimes X \otimes u$. In this tensor space we can define a product by $x y=a b X Y u v$ for $x=a X u$ and $y=b Y v$. Moreover an involution and a trace $T r$ can be given by $a X u \rightarrow \bar{a} X^{T} \bar{u}$ and $\operatorname{Tr}(a X u)=a \operatorname{tr}(X) I u$ respectively where $I$ is the $n \times n$ unit matrix. If $f(x, y, z)$ is a polynomial of $x, y$ and $z$, two sums $\Sigma f(x, y, z)$ and $\subseteq f(x, y, z)$ are defined as before.

\section{Exceptional Jordan algebra $J$.}

Let ( 5 be the Cayley algebra of non-split type (cf. Section 5 ) and let $M(3,(5)$ denote the matrix algebra over $\boldsymbol{R}$ of all $3 \times 3$ matrices with coefficients in $(\mathbb{5}$. Then we can define an isomorphism between two algebras $M(3, \mathbb{5})$ and $\mathbb{5} \otimes M(3, \boldsymbol{R})$ (resp. $M(3, \boldsymbol{R}) \otimes(\mathfrak{5})$ by $\left(e_{l} a_{\imath j}\right) \rightarrow e_{l} \otimes\left(a_{\imath j}\right)$ (resp. $\left.\left(a_{\imath \jmath}\right) \otimes e_{l}\right)$ for $e_{l} \in \mathbb{E}$ and $\left(a_{\imath j}\right) \in$ $M(3, \boldsymbol{R})$. Hence, if necessary, we shall identify these elements in the following way where we neglect the notation $\otimes$ :

$$
\left.\left(e_{l} a_{\imath \jmath}\right)=e_{l} A \text { (resp. } A e_{l}\right) \quad \text { for } \quad A=\left(a_{\imath \jmath}\right) \in M(3, \boldsymbol{R}) \text {; }
$$


especially, for the Jordan product $x \circ y=1 / 2(x y+y x)$ and the Lie product $[x, y]$ $=x y-y x$, we can write

$$
\begin{aligned}
& \left(e_{l} a_{\imath j}\right) \circ\left(e_{m} b_{\imath j}\right)=1 / 2\left(e_{l} e_{m} A B+e_{m} e_{l} B A\right), \\
& {\left[\left(e_{l} a_{\imath j}\right),\left(e_{m} b_{\imath j}\right)\right]=e_{l} e_{m} A B-e_{m} e_{l} B A,}
\end{aligned}
$$

where $e_{l}, e_{m} \in\left(5\right.$ and $A=\left(a_{\imath \jmath}\right), B=\left(b_{i \jmath}\right) \in M(3, R)$.

Let $J$ denote a vector space over $\boldsymbol{R}$ composed of all symmetric matrices in $M\left(3\right.$, (5) with respect to the involution: $a X \rightarrow \bar{a} X^{T}$. Then $J$ becomes an exceptional Jordan algebra by the Jordan product $\circ$. If an element $x \in J$ has the form

$$
\left(\begin{array}{ccc}
\xi_{1} & a_{3} & \bar{a}_{2} \\
\bar{a}_{3} & \xi_{2} & a_{1} \\
a_{2} & \bar{a}_{1} & \xi_{3}
\end{array}\right) \quad \xi_{i} \in R, \quad a_{i} \in \mathbb{}
$$

we can define the generic norm $(x, x)_{J}$ by $(x, x)_{J}=1 / 3\left(\xi_{1}{ }^{2}+\xi_{2}{ }^{2}+\xi_{3}{ }^{2}+2 a_{1} \bar{a}_{1}+\right.$ $\left.2 a_{2} \bar{a}_{2}+2 a_{3} \tilde{a}_{3}\right)$. If we put $L_{x}(y)=x \circ y$ and $A d(z) y=[z, y]$ for $x, y \in J$ and $z$ $(\in M(3,(5))$ with the skew-symmetric form, we have the following proposition.

Proposition 2.1. For $a X, b Y, c Z \in J$, we obtain that

(1) $(a X, b Y)_{J}=(a, b)(X, Y)$,

(2) $\Sigma(a X \circ b Y) \circ c Z-3 \varsigma(a X, b Y)_{J} c Z-6(a X \circ b Y, c Z)_{J} I=0$,

(3) $4\left[L_{a X}, L_{b Y}\right]=(X, Y) D_{a, b}+\operatorname{Ad}([a X, b Y]-\operatorname{Tr}[a X, b Y])$,

where $D_{a, b}(c Z)=\left(D_{a, b}(c)\right) Z$ and $\operatorname{Tr}(d W)=d \operatorname{tr}(W) I$ for $d W \in M(3),(5$.

Proof. (2) is the characteristic polynomial which is well known in the exceptional Jordan algebra $J$ (cf. [5]). For (3), in the case of the trace of $a X, b Y$ and $c Z$ being 0 , if we operate $4\left[L_{a X}, L_{b Y}\right]-(X, Y) D_{a, b}-\operatorname{Ad}([a X, b Y]-\operatorname{Tr}[a X$, $b Y])$ on $c Z$, we can have $-(a, b, c)(X Y Z+Z Y X+Y X Z+Z X Y-X Z Y-Y Z X-$ $3(X, Y) Z)$. This is identically 0 by $(2)$ in Proposition 1.2 and from the fact that the associator $(a, b, c)=0$ if one of the elements is real, i. e., $a=\bar{a}$ as an example.

Remark. Let $x$ be an element in $M(3,5)$ which is skew-symmetric and has the trace being 0 . Then, by (3) in Proposition 1.2, we can prove directly that $A d(x)$ is a derivation of $J$. Hence (3) in Proposition 2.1 can be considered a sharpened fact the operator $\left[L_{a X}, L_{b Y}\right]$ is a derivation in the Jordan algebra $J$. The automorphism group $A u t(J)$ of $J$ is the real simple Lie group of type $F_{4(-52)}$. If we use the Cayley algebra of split type in the above argument (cf. Section 5), we can have the exceptional Jordan algebra with the automorphism group of type $F_{4(4)}$. As for the type $F_{4(-20)}$, the corresponding algebra is given in Section 6. We note that Proposition 2.1 also holds for these algebras and moreover the identity (3) is true for other real simple Jordan algebras. 


\section{A construction of Lie algebras.}

We use the same notations as the last part of Section 1 . Let $\mathfrak{M}$ denote a vector space over $\boldsymbol{R}$ which is generated by all elements in $\mathfrak{U}^{(1)} \otimes M(n, \boldsymbol{R}) \otimes \mathfrak{U}^{(2)}$ with the trace $\operatorname{Tr}$ being 0 and the skew-symmetric form with respect to the involution $a X u \rightarrow \bar{a} X^{T} \bar{u}$. Let Der $\mathfrak{X}^{(1)}$ and $\operatorname{Der} \mathfrak{U}^{(2)}$ are the Lie algebras of all inner derivations of $\mathfrak{A}^{(1)}$ and $\mathfrak{A}^{(2)}$ respectively.

Let $L\left(\mathfrak{A}^{(1)}, M^{n}, \mathfrak{A}^{(2)}\right)$ be the vector space $\operatorname{Der} \mathfrak{U}^{(1)} \oplus \mathfrak{M} \oplus \operatorname{Der} \mathfrak{X}^{(2)}$ (direct sum) over $\boldsymbol{R}$. In this space we define an anti-commutative product $[$,$] in the follow-$ ing way:

(1) $\left[D^{(\imath)}, D^{(\jmath)}\right]=\left\{\begin{array}{cc}\text { the Lie product in } \operatorname{Der} \mathfrak{A}^{(\imath)} \quad(\imath=j), \\ 0 & (\imath \neq j),\end{array}\right.$

(2) $\left[D^{(1)}, a X u\right]=\left(D^{(1)} a\right) X u$ and $\left[D^{(2)}, a X u\right]=a X\left(D^{(2)} u\right)$,

(3) For $x=a X u, y=b Y v$ in $\mathfrak{M}$,

$$
[x, y]=(X, Y)(u, v) D_{a, b}+(x y-y x-\operatorname{Tr}(x y-y x))+(X, Y)(a, b) D_{u, v},
$$

where $D^{(i)}, \imath=1,2$, are elements in $\operatorname{Der} \mathfrak{U}^{(i)}$.

THEOREM 3.1. $L\left(\mathfrak{U}^{(1)}, M^{n}, \mathfrak{A}^{(2)}\right)$ becomes a real Lie algebra by the anti-commutative product [,] where, if $\mathfrak{U}^{(1)}$ or $\mathfrak{U}^{(2)}$ is a Cayley algebra, $n=1$ or 3 .

Remark. $L\left(\mathfrak{U}^{(1)}, M^{n}, \mathfrak{U}^{(2)}\right)$ is isomorphic to $L\left(\mathfrak{H}^{(2)}, M^{n}, \mathfrak{U}^{(1)}\right)$. If $\mathfrak{A}^{(1)}=\boldsymbol{R}$, $L\left(\boldsymbol{R}, M^{n}, \mathfrak{Y}^{(2)}\right)$ is a compact simple Lie algebra of type $B_{(n-1) / 2}$ (or $D_{n / 2}$ ), $A_{n-1}$ or $C_{n}$ according as $\mathfrak{U}^{(2)}$ is $\boldsymbol{R}, \boldsymbol{C}$ or $\boldsymbol{Q}$ where $\boldsymbol{C}$ and $\boldsymbol{Q}$ are the field of complex and quarternion numbers with the non-split type respectively. If $\mathfrak{A}^{(1)}=\complement$, $L\left(\sqrt{5}, M^{3}, \mathfrak{A}^{(2)}\right)$ is a compact simple Lie algebra of type $F_{4}, E_{6}, E_{7}$ or $E_{8}$ according as $\mathfrak{A}^{(2)}$ is $\boldsymbol{R}, \boldsymbol{C}, \boldsymbol{Q}$ or $(5$. We note that Theorem 3.1 also holds for all composition algebras with the split type. In this section we shall prove only Theorem 3.1 and, in Section 4, establish an isomorphism between the above models and Tits' Lie algebras instead of the proof for the compactness and the simpleness of $L\left(\mathfrak{Y}^{(1)}, M^{n}, \mathfrak{U}^{(2)}\right)$. Then we shall have a new viewpoint for Tits' construction.

Proof of Theorem 3.1. In the case of $n=3$ and $\mathfrak{X}^{(1)}$ being the Cayley algebra with the non-split or the split type, we shall prove that the Jacobi's 1dentity holds for $x, y, z \in \mathfrak{M}$. In other cases, we can prove easily it by Lemma 1.1 and the definition of $L\left(\mathfrak{X}^{(1)}, M^{n}, \mathfrak{A}^{(2)}\right)$

For $x=a X u, y=b Y v, z=c Z w \in \mathfrak{M}$, from long calculations, we sbtain that

$$
\begin{aligned}
& {[[x, y], z]+[[y, z], x]+[[z, x], y]=(\mathrm{i})+(\mathrm{ii})+(\mathrm{iii})+(\mathrm{iv})+(\mathrm{v}): } \\
&(\mathrm{i})=(X Y, Z)(u v, w) \subseteq D_{a b, c}+(X Y, Z)(a b, c) \subseteq D_{u v, w} \\
&-(Y X, Z)(v u, w) \subseteq D_{b a, c}-(Y X, Z)(b a, c) \subseteq D_{v u, w} \\
&= 0 \quad \text { by Lemma } 1.1,
\end{aligned}
$$




$$
\begin{aligned}
\text { (ii) }= & (a, b, c)\{\Sigma X Y Z(u v) w\}+\{\Sigma a(b c) X Y Z\}(u, v, w), \\
\text { (iii) }= & -(a, b, c)\{\Sigma \operatorname{tr}(X Y Z) I(u v) w\}-\{\Sigma a(b c) \operatorname{tr}(X Y Z) I\}(u, v, w), \\
\text { (iv) }= & \varsigma\{-[a b, c](X, Y) Z(u v) w-c(a b)(X, Y) Z[u v, w] \\
& +[b a, c](X, Y) Z(v u) w+c(b a)(X, Y) Z[v u, w]\}
\end{aligned}
$$

and

$$
\begin{aligned}
(\mathrm{v})= & \boldsymbol{S}(X, Y)(u, v)[[a, b], c] Z w-3(a, b, c) \subseteq(X, Y)(u, v) Z w \\
& +\subseteq(X, Y)(a, b) c Z[[u, v], w]-3\{\subseteq(X, Y)(a, b) c Z\}(u, v, w),
\end{aligned}
$$

where for (ii) and (iii) the definition $a(b c)=(a b) c-(a, b, c)$ is used repeatedly and for $(\mathrm{v})$ we use $D_{a, b}(c)=[[a, b], c]-3(a, b, c)$.

Here our proof is divided into the four cases:

(case 1) $a, b, c, u, v, w$ are purely imaginary numbers,

(case 2) $a, b, c$ are real numbers,

(case 3) $a, b$ are real numbers and $c$ is a purely imaginary number,

(case 4) $a$ is a real number and $b, c$ are purely imaginary numbers,

where that $a \in \mathfrak{A}$ is a real number means $a=\bar{a}$ and that $a$ is a purely imaginary number means $a=-\bar{a}$.

(case 1) By three identities $\Sigma X Y Z(u v) w=\Sigma(X u \circ Y v) \circ Z w+1 / 2\{\Sigma X Y Z$ $(u, v, w)\}, \Sigma a(b c) X Y Z=\Sigma(a X \circ b Y) \circ c Z-1 / 2\{\Sigma(a, b, c) X Y Z\}$ and $\operatorname{Tr}((X u \circ Y v) \circ Z w)$ $=(X u \circ Y v, Z w)_{J} I$, we obtain

$$
\begin{aligned}
& \text { (ii) }=(a, b, c)\{\Sigma(X u \circ Y v) \circ Z w\}+\{\Sigma(a X \circ b Y) \circ c Z\}(u, v, w), \\
& \text { (iii) }=-(a, b, c)\left\{\Sigma(X u \circ Y v, Z w)_{J} I\right\}-\left\{\Sigma(a X \circ b Y, c Z)_{J} I\right\}(u, v, w) .
\end{aligned}
$$

And, by $a b+b a=2(a, b)$ and $[[a, b], c]=2[a b, c]$, we have

$$
\text { (iv) }=-\mathfrak{S}(X, Y)(u, v)[[a, b], c] Z w-\mathfrak{S}(X, Y)(a, b) c Z[[u, v], w] \text {. }
$$

Hence the total sum (ii) $+($ iii $)+($ iv $)+(v)$ is

$$
\begin{aligned}
& (a, b, c)\left\{\Sigma(X u \circ Y v) \circ Z w-3 \varsigma(X u, Y v)_{J} Z w-6(X u \circ Y v, Z w)_{J} I\right\} \\
& \quad+\left\{\Sigma(a X \circ b Y) \circ c Z-3 \varsigma(a X, b Y)_{J} c Z-6(a X \circ b Y, c Z)_{J} I\right\}(u, v, w) .
\end{aligned}
$$

This is identically 0 by (2) in Proposition 2.1.

(case 2) The total sum gets

$$
a b c\{\Sigma X Y Z-3 \varsigma(X, Y) Z-\Sigma \operatorname{tr}(X Y Z) I\}(u, v, w) .
$$

This is also 0 by (1) in Proposition 1.2.

(case 3 ) The total sum becomes

$$
\begin{aligned}
a b c & \{\Sigma X Y Z-3(X, Y) Z-\Sigma \operatorname{tr}(X Y Z) I\}(u, v, w)-a b c \varsigma(X, Y) Z[[u, v], w] \\
& +a b c(X, Y) Z[[u, v], w] .
\end{aligned}
$$


If $u, v, w$ are purely imaginary numbers, the sum is 0 by (4) in Proposition 1.2 and $(X, Z)=(Y, Z)=0$. If not so, it gets 0 obviously.

(case 4) If $u$ is a real number, the sum is 0 evidently. Hence we can assume that $u$ is a purely imaginary number. If $v$ or $w$ is a real number, the sum becomes

$$
\begin{aligned}
a\{- & {[b, c](X, Y) Z[u, v] w+[b, c](Z, X) Y[w, u] v-b c(Y, Z) X[v w, u] } \\
& +c b(Y, Z) X[w v, u]\} .
\end{aligned}
$$

Especially, in the case of $v$ and $w$ being real numbers, it is 0 because all $[u, v]$, $[w, u],[v w, u]$ are 0 . If $v$ is a real number and $w$ is a purely imaginary number, $X$ and $Y$ are symmetric matrices and $Z$ is a skew-symmetric matrix. Hence it is 0 because $[u, v],(X, Z)$ and $(Y, Z)$ are 0 . If $v$ is a purely imaginary number and $w$ is a real number, $X$ and $Z$ are symmetric matrices and $Y$ is a skewsymmetric matrix. Hence it is also 0 because $[w, u],(X, Y)$ and $(Y, Z)$ are 0 . Finally, if $v$ and $w$ are purely imaginary numbers, $Y$ and $Z$ are skew-symmetric matrices and $X$ is a symmetric matrix with the trace being 0 . Then, by $(X, Y)$ $=(X, Z)=0$ and $v w+w v=2(v, w)$, the sum can be rewritten as

$$
\{\Sigma a(b c) X Y Z-3(Y, Z)(b, c) a X-\Sigma a(b c) \operatorname{tr}(X Y Z) I\}(u, v, w) .
$$

Furthermore, from (3) in Proposition 1.2, it becomes

$$
a(b, c)\{2(X Y Z+Y Z X+Y X Z)-3(Y, Z) X-6 \operatorname{tr}(X Y Z) I\}(u, v, w)
$$

and at last 0 identically.

\section{An isomorphism to Tits' Lie algebras.}

We shall establish an isomorphism between our Lie algebra $L\left(\mathfrak{A}^{(1)}, M^{3}, \mathfrak{A}^{(2)}\right)$ and one of Tits' Lie algebras. Let $\mathfrak{A}_{0}$ (resp. $J_{0}$ ) denote a vector space over $\boldsymbol{R}$ composed of all elements in an composition algebra $\mathfrak{A}$ (resp. the exceptional Jordan algebra $J$ in Section 2) which are orthogonal to the unit element with respect to the inner product $(),\left(\operatorname{resp} .(,)_{J}\right)$. Let $\operatorname{Der} J$ be the Lie algebra of all inner derivations of $J$, i.e., $\left[L_{x}, L_{y}\right]$ where $x, y \in J_{0}$. Now we give an anticommutative product $[$,$] after Tits in the vector space \operatorname{Der} J \oplus J_{0} \otimes \mathfrak{A}_{0} \oplus D e r \mathfrak{A}$ over $\boldsymbol{R}$ such that

(1) $\left[D_{1}, D_{2}\right]=\left\{\begin{array}{r}\text { the original Lie product for } D_{1}, D_{2} \in \operatorname{Der} J \text { or } \\ D_{1}, D_{2} \in \operatorname{Der} \mathfrak{A}, \\ 0 \quad \text { for } D_{1} \in \operatorname{Der} J \text { and } D_{2} \in \operatorname{Der} \mathfrak{A},\end{array}\right.$

(2) $\left[D_{1}, x \otimes a\right]=D_{1} x \otimes a$ and $\left[D_{2}, x \otimes a\right]=x \otimes D_{2} a$ for $D_{1} \in \operatorname{Der} J, D_{2} \in \operatorname{Der} \mathfrak{A}$ and $x \otimes a \in J_{0} \otimes \mathfrak{A}_{0}$,

(3) for $x \otimes a, y \otimes b \in J_{0} \otimes \mathfrak{A}_{0}$,

$$
[x \otimes a, y \otimes b]=(a, b)\left[L_{x}, L_{y}\right]+x * y \otimes a * b+1 / 4(x, y)_{J} D_{a, b},
$$


where $x * y=x \circ y-(x, y)_{J} I$ and $a * b=a b-(a, b) e_{0}$ ( $I$ is the $3 \times 3$ unit matrix and $e_{0}$ is the unit element in $\left.\mathfrak{U}\right)$. This algebra becomes a real simple Lie algebra. The above definition is taken from H. Freudenthal [2] and it is similar to our construction except (3).

Let $\mathfrak{M}_{\text {re }}$ and $\mathfrak{M}_{\mathrm{im}}$ be vector subspaces of $\mathfrak{M}$ which are composed of all elements $a X u$ where each $u$ is a real number or a purely imaginary number respectively. Then $\mathfrak{M}=\mathfrak{M}_{\mathrm{re}} \oplus \mathfrak{M}_{\mathrm{im}}$. Now we give an isomorphism $f$ between the two Lie algebras:

$$
f \downarrow_{\left(D^{(1)}+u A d(a X)\right)+2 b Y \otimes v+D^{(2)} \in D \operatorname{Der} J \oplus J_{0} \otimes \mathfrak{A}_{0}^{(2)} \oplus \operatorname{Der} \mathfrak{A}^{(2)}}
$$

where $D^{(1)}(c Z)=\left(D^{(1)} c\right) Z$ for $c Z \in J$ and $\mathfrak{X}^{(1)}=(\mathfrak{c}$. By Remark of Proposition 2.1, we can see that the definition of $f$ is well-defined. And that $f$ is a homomorphism can be proved by $(a X, b Y)_{J}=(a, b)(X, Y)$ and the following proposition.

Proposition 4.1. If $a X$ and $b Y$ are skew-symmetric matrices in $M(3$, (5) with the trace being 0 , we have

(1) $[A d(a X), A d(b Y)]=(X, Y) D_{a, b}+\operatorname{Ad}([a X, b Y]-\operatorname{Tr}[a X, b Y])$,

(2) $[D, A d(a X)]=A d((D a) X)$ for $D \in \operatorname{Der} \mathfrak{A}^{(1)}$.

(1) is obtained from (4) in Proposition 1.2. This proposition also holds for other real simple Jordan algebras.

Theorem 4.2. The Lie algebra $L\left(\mathfrak{2}^{(1)}, M^{3}, \mathfrak{A}^{(2)}\right)$ is isomorphic to one of Tits' Lie algebras.

Remark. If we use two matrix algebras $M^{3}, M^{3}{ }_{3}$, composition algebras of split or non-split type and the exceptional Jordan algebras (cf. Section 2,6), we can construct all Tits' Lie algebras by the isomorphism $f$.

We also know that E. B. Vinberg [7] gave a construction of exceptional Lie algebras. Noting that $(a ; b)=1 / 2(a \bar{b}+b \bar{a})$ is used in [7] as the inner product of composition algebras, we can give easily an isomorphism between our and Vinberg's models. So we can say that these algebras stand at the same standpoint in essential.

\section{Composition algebras.}

First we give a basis explicitely in the Cayley algebra $\boldsymbol{s}$ of non-split type over $\boldsymbol{R}$ as follows:

the basis: $e_{0}, e_{1}, \cdots, e_{7}$;

the rule of the product:

$$
e_{1} e_{2}=e_{3}, \quad e_{1} e_{4}=e_{5}, \quad e_{6} e_{7}=e_{1}, \quad e_{2} e_{5}=e_{7}, \quad e_{3} e_{4}=e_{7}, \quad e_{3} e_{5}=e_{6}, \quad e_{6} e_{4}=e_{2},
$$


$e_{\imath} e_{\jmath}=-e_{j} e_{\imath} \quad(\imath, j \geqq 1$ and $\imath \neq j), \quad e_{\imath} e_{\imath}=-e_{0} \quad(i \geqq 1)$,

$e_{0}$ is the unit element;

the conjugate operator - : $e_{0} \rightarrow e_{0}, e_{2} \rightarrow-e_{2}(1 \leqq \imath \leqq 7)$.

Then in the Cayley algebra $(5$ we can realize $\boldsymbol{R}, \boldsymbol{C}$ or $\boldsymbol{Q}$ as subalgebras generated by $\left\{e_{0}\right\},\left\{e_{0}, e_{1}\right\}$ or $\left\{e_{0}, e_{1}, e_{2}, e_{3}\right\}$ respectively. In each composition algebra (except $\boldsymbol{R}$ ) of non-split type, we have essentially one kind of involutive automorphism $\alpha$ (cf. K. Atsuyama [1]) defined by

$$
\begin{gathered}
\boldsymbol{Q} \\
\alpha \downarrow_{e_{0},}^{e_{0},} \quad e_{1} \quad e_{0}, e_{1}, \quad e_{2}, \quad e_{3}, e_{1},-e_{0}, \cdots,-e_{3}, \quad e_{0}, \cdots, e_{3},-e_{4}, \cdots,-e_{7},
\end{gathered}
$$

This $\alpha$ makes the inner product $(a, b)$ and the unit element $e_{0}$ invariant.

Now we consider the Cayley algebra $(5$ as an example. The $(-1)$-eigenspace for the involutive automorphism $\alpha$ is a vector space of the basis $\left\{e_{4}, e_{5}, e_{6}, e_{7}\right\}$ Then we can obtain the other real Cayley algebra $\S_{\alpha}$ with the split type by the complexification of this (-1)-eigenspace. And the basis of $\boldsymbol{5}_{\alpha}$ is given by

$$
c_{0}, e_{1}, e_{2}, e_{3}, \boldsymbol{i} e_{4}, \boldsymbol{i} e_{5}, \boldsymbol{i} e_{6}, \boldsymbol{i} e_{7} \quad\left(\boldsymbol{i}^{2}=-1\right) .
$$

In this way we can get all composition algebras of split type, i.e., $\boldsymbol{C}_{\alpha}, \boldsymbol{Q}_{\alpha}$ and $\mathfrak{L}_{\alpha}$. Each of these algebras has the conjugation defined by in $\mathfrak{E}_{\alpha}$, for example, $e_{0} \rightarrow e_{0}, e_{i} \rightarrow-e_{\imath}(1 \leqq \imath \leqq 3)$ and $\boldsymbol{i} e_{i} \rightarrow-\boldsymbol{i} e_{\imath}(4 \leqq i \leqq 7)$.

A basis in $\operatorname{Der} \mathfrak{A}$ is also given according as $\mathfrak{A}$ is $\boldsymbol{R}, \boldsymbol{C}, \boldsymbol{Q}$ or $\mathbb{E}$ by $\{0\},\{0\}$, $\left\{D_{e \imath, e\}}\right\}$ with $(\imath, j)=(1,2),(2,3)$ and $(3,1)$ or $\left\{D_{e \imath, e j}\right\}$ with $(i, j)=(1,2),(2,3)$, $(3,1),(4,5),(6,4),(4,7),(1,4),(5,1),(1,6),(7,1),(4,2),(2,6),(7,2)$ and $(3,4)$. And the dimension of the $(-1)$-eigenspace for an involutive automorphism: $D_{a, b} \rightarrow D_{\alpha a, \alpha b}$ in Der $\mathfrak{A}$ is $0,0,2$ or 8 respectively.

\section{Matrix algebras.}

The $n \times n$ real matrix algebra $M(n, \boldsymbol{R})\left(=M^{n}\right)$ has two kinds of involutive automorphisms $\beta, \gamma$. $\beta$ is defined for any natural number $n=p+q$ such that

$$
\beta: \stackrel{p}{q}\left(\begin{array}{c:c}
A & q \\
\hdashline C & D
\end{array}\right) \rightarrow\left(\begin{array}{cc}
A & B \\
-C & D
\end{array}\right) .
$$

It makes the inner product $(X, Y)$ and the unit matrix $I$ invariant. Note that $\beta$ depends on $p$ (or $q$ ). Especially, if $n=3$, there exists only one involutive automorphism (up to conjugation) corresponding to $p=1$ and $q=2$. The other kind of involutive automorphism $\gamma$ is defined only for an even number $n=2 p$ such that 


$$
\gamma:\left(A_{\imath j}\right) \longrightarrow\left(\gamma_{0} A_{\imath j}\right) \quad \text { for } \quad A_{\imath \jmath} \in M(2, \boldsymbol{R}) \text { and } 1 \leqq \imath, \jmath \leqq p,
$$

where $\gamma_{0}$ is an involutive automorphism in $M(2, \boldsymbol{R})$ which is given by $\left(\begin{array}{ll}a & b \\ c & d\end{array}\right) \rightarrow\left(\begin{array}{rr}d & -c \\ -b & a\end{array}\right)$ for $a, b, c, d \in \boldsymbol{R} . \quad \gamma$ also makes the inner product $(X, Y)$ and the unit matrix $I$ invariant. We note that $\gamma_{0}$ relates to an involutive automorphism in $\boldsymbol{Q}_{\alpha}$ and, if $p$ and $q$ are even number, $\beta$ and $\gamma$ are commutative, i.e., $\beta \gamma$ is an involutive automorphism again.

Next we construct real matrix algebras $M^{n}{ }_{\beta}$ and $M_{\gamma}^{n}$ corresponding to $\beta$ and $\gamma$ respectively. $M_{\beta}^{n}$ is the matrix algebra with the basis obtained by the complexification of the $(-1)$-eigenspace for $\beta$ : The forms of the basis are

$$
\left(\begin{array}{ll}
A & 0 \\
0 & D
\end{array}\right) \quad \text { or } \quad \boldsymbol{i}\left(\begin{array}{ll}
0 & B \\
C & 0
\end{array}\right) \quad\left(\boldsymbol{i}^{2}=-1\right) .
$$

$M^{n}$ is the matrix algebra with the basis obtained by the complexification of the $(-1)$-eigenspace for $\gamma$ : The forms of the basis are

$$
\left(A_{\imath j}\right) \text { or } \boldsymbol{i}\left(B_{\imath j}\right) \quad\left(\boldsymbol{i}^{2}=-1\right),
$$

where $A_{\imath \jmath}=\left(\begin{array}{rr}a & b \\ -b & a\end{array}\right), B_{\imath \jmath}=\left(\begin{array}{rr}d & c \\ c & -d\end{array}\right) \subseteq M(2, \boldsymbol{R})$ and $1 \leqq \imath, \jmath \leqq p$.

Remark. Here we can give an exceptional Jordan algebra with the automorphism group of type $F_{4(-20)}$ by making use of $\beta$ for $n=3$. In fact, let $J$ be the exceptional Jordan algebra of type $F_{4(-52)}$ (in Section 2). Then the algebra can be obtained in the similar way above by the complexification of the $(-1)$ eigenspace for an automorphism: $a X \rightarrow a \beta X$ in $J$.

\section{Real forms.}

We can see later that any involutive automorphism in each compact simple Lie algebra can be made of three kinds of involutive automorphisms $\alpha, \beta$ and $\gamma$ in the composition or the matrix algebras. First we extend these maps to involutive automorphisms in the Lie algebra $L\left(\mathfrak{A}^{(1)}, M^{n}, \mathfrak{A}^{(2)}\right)$ by the following way :

$$
\begin{aligned}
& \alpha_{1}: D_{a, b}+c X d+D_{u, v} \longrightarrow D_{\alpha a, \alpha b}+(\alpha c) X d+D_{u, v}, \\
& \alpha_{2}: D_{a, b}+c X d+D_{u, v} \longrightarrow D_{a, b}+c X(\alpha d)+D_{\alpha u, \alpha v}, \\
& \beta: D_{a, b}+c X d+D_{u, v} \longrightarrow D_{a, b}+c(\beta X) d+D_{u, v}
\end{aligned}
$$

and $\gamma$ is defined by the same way as $\beta$. In this definition we use the same notations for the extended involutive automorphisms. Then we can see easily that $\alpha_{\imath}, \beta, \gamma, \alpha_{1} \alpha_{2}, \alpha_{1} \alpha_{2} \beta, \alpha_{i} \beta$ and $\alpha_{\imath} \gamma(\imath=1,2)$ are again involutive automorphisms but $\beta \gamma$ and $\alpha_{\imath} \beta \gamma(\imath=1,2)$ are involutive under the condition that both $p$ and $q$ 
$(n=p+q)$ of $\beta$ are even number. Note that $\gamma, \beta \gamma, \alpha_{\imath} \gamma$ and $\alpha_{i} \beta \gamma \quad(l=1,2)$ exist only if $n$ (of $M^{n}$ ) is even number.

Now we can give all the real simple Lie algebras in the following table. We write in (a) compact simple Lie algebras, in (b) those involutive automorphisms, in (c) the dimension of the (-1)-eigenspace for each involutive automorphism, in (d) and (e) the types of the irreducible Riemannian symmetric space and the models of the non-compact simple Lie algebra respectively corresponding to each involutive automorphism (cf. S. Helgason [3]) and in (f) the types of exceptional Lie algebras. We can know that the models in (e) are real simple Lie algebras by the following commutative diagrams (up to isomorphism); in the case of $L\left(M_{\beta}^{n}, \boldsymbol{C}_{\alpha}\right)$, for example,

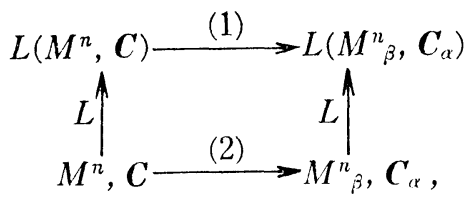

where (1) is the duality by $\alpha \beta$, 1. e., the complexification of the (-1)-eigenspace of $\alpha \beta$, (2) is the duality by $\alpha$ and $\beta$ for $C$ and $M^{n}$ respectively and $L$ is the our construction of Lie algebras.

We omit $\boldsymbol{R}$ from the notation $L\left(\mathfrak{H}^{(1)}, M^{n}, \mathfrak{X}^{(2)}\right)$ if one of these algebras is $\boldsymbol{R}$ and also omit the suffix $\imath$ of $\alpha$, unless the confusion does not occur.

Table Real Simple Lie Algebras

\begin{tabular}{|c|c|c|c|c|}
\hline (a) & (b) & (c) & (d) & (e) \\
\hline \multirow[t]{3}{*}{$A_{n-1} L\left(M^{n}, \boldsymbol{C}\right)$} & $\alpha, \alpha \beta$ & $(n-1)(n+2) / 2$ & $A \mathrm{I}$ & \multirow{3}{*}{$\begin{array}{l}L\left(M^{n}, \boldsymbol{C}_{\alpha}\right) \\
L\left(M^{n}{ }_{\beta}, \boldsymbol{C}_{\alpha}\right) \\
L\left(M^{n}{ }_{\gamma}, \boldsymbol{C}_{\alpha}\right) \\
L\left(M^{n}{ }_{\beta \gamma}, \boldsymbol{C}_{\alpha}\right) \\
L\left(M^{n}{ }^{\beta}, \boldsymbol{C}\right) \\
L\left(M^{n}{ }_{\gamma}, \boldsymbol{C}\right) \\
L\left(M^{n}{ }_{\beta \gamma}, \boldsymbol{C}\right)\end{array}$} \\
\hline & $\alpha \gamma, \alpha \beta \gamma$ & $(n-2)(n+1) / 2$ & $A \Pi$ & \\
\hline & $\beta, \gamma, \beta \gamma$ & $2 p q$ & $A$ III & \\
\hline$B_{(n-1) / 2} L\left(M^{n}\right)$ & $\beta$ & $p q$ & $B \mathrm{I}, B \mathrm{II}$ & $L\left(M_{\beta}^{n}\right)$ \\
\hline \multirow[t]{2}{*}{$C_{n} L\left(M^{n}, \boldsymbol{Q}\right)$} & $\alpha, \alpha \beta, \gamma, \beta \gamma$ & $n(n+1)$ & $C \mathrm{I}$ & $\begin{array}{l}L\left(M^{n}, \boldsymbol{Q}_{\alpha}\right) \\
L\left(M^{n}{ }_{\beta}, \boldsymbol{Q}_{\alpha}\right) \\
L\left(M^{n}{ }_{\gamma}, \boldsymbol{Q}\right) \\
L\left(M^{n}{ }_{\beta \gamma}, \boldsymbol{Q}\right)\end{array}$ \\
\hline & $\beta, \alpha \gamma, \alpha \beta \gamma$ & $4 p q$ & $C$ II & $\begin{array}{l}L\left(M^{n}{ }_{\beta}, \boldsymbol{Q}\right) \\
L\left(M^{n}{ }^{\gamma}, \boldsymbol{Q}_{\alpha}\right) \\
L\left(M^{n}{ }_{\beta \gamma}, \boldsymbol{Q}_{\alpha}\right)\end{array}$ \\
\hline
\end{tabular}


Table Contınued

\begin{tabular}{|c|c|c|c|c|c|}
\hline (a) & (b) & (c) & (d) & (e) & (f) \\
\hline$D_{n / 2} L\left(M^{n}\right)$ & $\begin{array}{c}\beta \\
\gamma \gamma\end{array}$ & $\begin{array}{c}p q \\
n(n-2) / 4\end{array}$ & $\begin{array}{l}D \mathrm{I}, D \mathrm{II} \\
D \mathrm{III}\end{array}$ & $\begin{array}{l}L\left(M_{\beta}^{n}\right) \\
L\left(M_{\gamma}^{n}\right) \\
L\left(M_{\beta \gamma}^{n}\right)\end{array}$ & \\
\hline \multirow[t]{4}{*}{$E_{6} L\left(\varsigma, M^{3}, \boldsymbol{C}\right)$} & $\alpha_{1} \alpha_{2}, \alpha_{1} \alpha_{2} \beta$ & 42 & $E \mathbf{I}$ & $\begin{array}{l}L\left(\boldsymbol{(}_{\alpha}, M^{3}, \boldsymbol{C}_{\alpha}\right) \\
L\left(\boldsymbol{\S}_{\alpha}, M_{\beta}^{3}, \boldsymbol{C}_{\alpha}\right)\end{array}$ & $E_{6(6)}$ \\
\hline & $\alpha_{1}, \alpha_{1} \beta$ & 40 & $E \Pi$ & $\begin{array}{l}L\left(\mathbb{(}_{\alpha}, M^{3}, \boldsymbol{C}\right) \\
L\left(\S_{\alpha}, M^{3}{ }_{\beta}, \boldsymbol{C}\right)\end{array}$ & $E_{6(2)}$ \\
\hline & $\beta$ & 32 & $E$ III & $L\left(\mathbb{B}, M^{3}{ }_{\beta}, \boldsymbol{C}\right)$ & $E_{6(-14)}$ \\
\hline & $\alpha_{2}, \alpha_{2} \beta$ & 26 & $E \mathrm{IV}$ & $\begin{array}{l}L\left(\mathbb{(}, M^{3}, \boldsymbol{C}_{\alpha}\right) \\
L\left(\mathbb{(}, M^{3}{ }_{\beta}, \boldsymbol{C}_{\alpha}\right)\end{array}$ & $E_{6(-26)}$ \\
\hline \multirow[t]{3}{*}{$E_{7} L\left(\varsigma, M^{3}, \boldsymbol{Q}\right)$} & $\alpha_{1} \alpha_{2}, \alpha_{1} \alpha_{2} \beta$ & 70 & $E \mathrm{~V}$ & $\begin{array}{l}L\left(\S_{\alpha}, M^{3}, \boldsymbol{Q}_{\alpha}\right) \\
L\left(\S_{\alpha}, M_{\beta}^{3}, \boldsymbol{Q}_{\alpha}\right)\end{array}$ & $E_{7(7)}$ \\
\hline & $\alpha_{1}, \alpha_{1} \beta, \beta$ & 64 & $E \mathrm{VI}$ & $\begin{array}{l}L\left(\mathbb{S}_{a}, M^{3}, \boldsymbol{Q}\right) \\
L\left(\mathbb{S}_{\alpha}, M^{3}{ }_{\beta}, \boldsymbol{Q}\right) \\
L\left(\mathbb{S}, M^{3}{ }_{\beta}, \boldsymbol{Q}\right)\end{array}$ & $E_{7(-5)}$ \\
\hline & $\alpha_{2}, \alpha_{2} \beta$ & 54 & $E \mathrm{VII}$ & $\begin{array}{l}L\left(\mathbb{(}, M^{3}, \boldsymbol{Q}_{\alpha}\right) \\
L\left(\mathbb{(}, M^{3}{ }_{\beta}, \boldsymbol{Q}_{\alpha}\right)\end{array}$ & $E_{7(-25)}$ \\
\hline \multirow[t]{2}{*}{$E_{8} L\left(\mathbb{5}, M^{3}, \mathbb{5}\right)$} & $\begin{array}{l}\alpha_{1} \alpha_{2}, \beta \\
\alpha_{1} \alpha_{2} \beta\end{array}$ & 128 & $E \mathrm{VIII}$ & 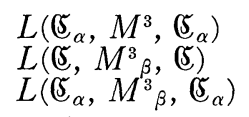 & $E_{8(8)}$ \\
\hline & $\begin{array}{l}\alpha_{1}, \alpha_{2}, \alpha_{1} \beta \\
\alpha_{2} \beta\end{array}$ & 112 & $E \mathrm{IX}$ & 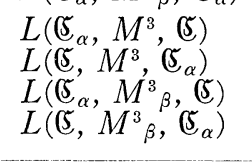 & $E_{8(-24)}$ \\
\hline \multirow[t]{2}{*}{$F_{4} L\left(8, M^{3}\right)$} & $\alpha_{1}, \alpha_{1} \beta$ & 28 & $F \mathrm{I}$ & $\begin{array}{l}L\left(\varsigma_{\alpha}, M^{3}\right) \\
L\left(\aleph_{\alpha}, M_{\beta}^{3}\right)\end{array}$ & $F_{4(4)}$ \\
\hline & $\beta$ & 16 & $F \amalg$ & $L\left(\mathbb{B}, M_{\beta}^{3}\right)$ & $F_{4(-20)}$ \\
\hline$G_{2} L($ (5) & $\alpha$ & 8 & $G$ & $L\left(\S_{\alpha}\right)$ & $G_{2(2)}$ \\
\hline
\end{tabular}

\section{REFERENCES}

[1] K. Atsuyama, Symmetric spaces derived from algebras, Kodai Math. J. 3 (1980), 358-363.

[2] H. Freudenthal, Lie groups in the foundations of geometry, Advances in Math. 1 (1965), 145-190.

[3] S. Helgason, Diffential Geometry, Lie groups, and Symmetric Spaces, Academic Press, New York, 1978.

[4] N. JACOBSon, Exceptional Lie algebras, Marcel Dekker, New York, 1971.

[5] R.D. Schafer, An introduction to nonassociative algebras, Academic Press, New York, 1966. 
[6] J. Tirs, Algèbres alternatives, algèbres de Jordan et algèbres de Lie exceptıonnelles I. Construction, Nederl. Akad. Weten. Proc. Ser. A, 69 (1966), 223-237.

[7] E.B. ViNBERG, Construction of the exceptional simple lie algebras (Russian), Trudy Sem. Vektor. Tenzor. Anal. 13 (1966), 7-9.

Kumamoto Instinete of Technology

IKEDA, KUMAMOTO 860

JAPAN 
